UCRL JC- 124437

PREPRINT

\title{
Recent Results from the EBIT and Super EBIT at Lawrence Livermore National Laboratory
}

\author{
R. E. Marrs
}

Prepared for Submittal to

Proceeding of Eighth International Conference on Physics of Highly Charged Ions

Omiya, Saitama, Japan, September 23-26, 1996

October 7, 1996

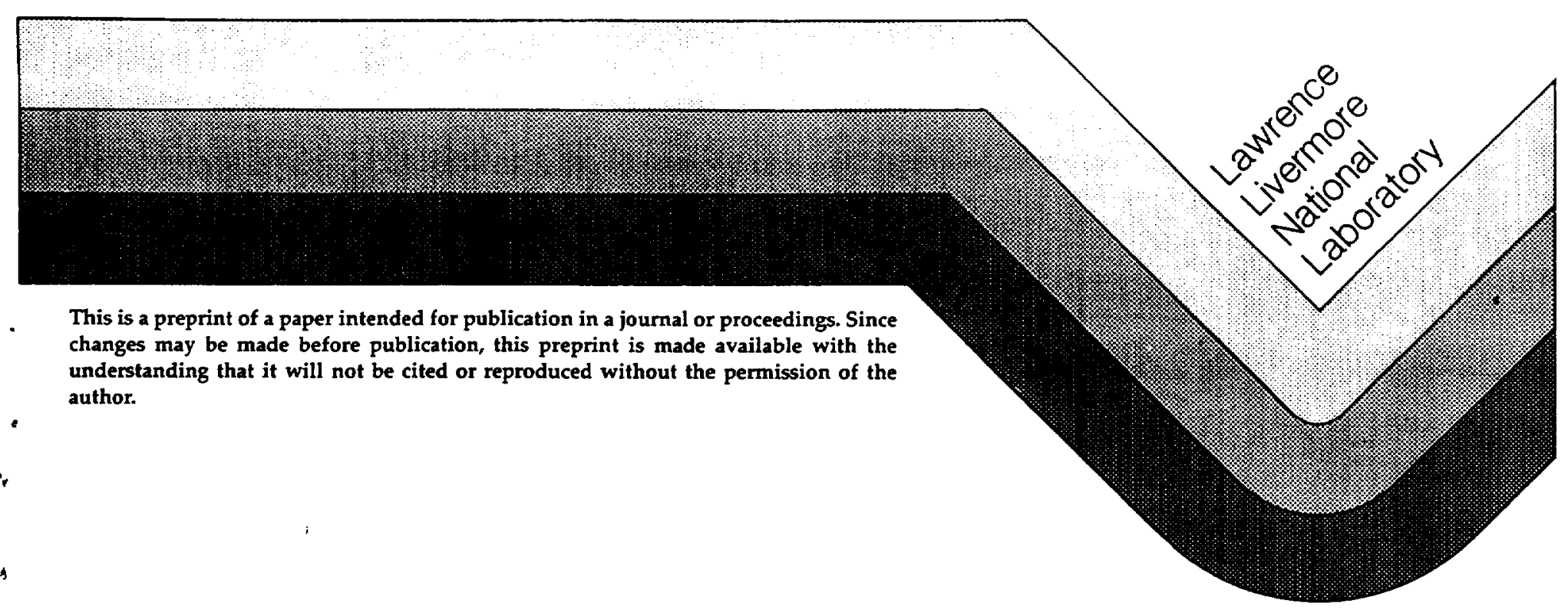




\section{DISCLAIMER}

This document was prepared as an account of work sponsored by an agency of the United States Government. Neither the United States Government nor the University of California nor any of their employees, makes any warranty, express or implied, or assumes any legal liability or responsibility for the accuracy, completeness, or usefulness of any information, apparatus, product, or process

disclosed, or represents that its use would not infringe privately owned rights. Reference herein to any specific commercial product, process, or service by trade name, trademark, manufacturer, or otherwise, does not necessarily constitute or imply its endorsement, recommendation, or favoring by the United States Government or the University of California. The views and opinions of authors expressed herein do not necessarily state or reflect those of the United States Government or the University of California, and shall not be used for advertising or product endorsement purposes. 


\title{
Recent Results from the EBIT and Super EBIT at Lawrence Livermore National Laboratory
}

\author{
R. E. Marrs \\ Lawrence Livermore National Laboratory, Livermore, California 94551
}

\begin{abstract}
The electron beam ion trap (EBIT), and the higher-energy Super EBIT at Lawrence Livermore National Laboratory can produce any highly charged ion. These highly charged ions are used in a variety of research programs. Recent results from four different experiments are reviewed here. $\mathrm{K}$-shell ionization cross sections have been measured for the hydrogenlike ions of several elements, and L-shell ionization cross sections have been measured for uranium ions. A measurement of the ground-state hyperfine transition in hydrogenlike ${ }^{165} \mathrm{Ho}^{66+}$ is notable because of the complete absence of Doppler shifts. A cryogenic Penning trap, injected with EBIT ions, has been used to observe a single highly charged ion as it recombines by sequential electron capture from $\mathrm{H}_{2}$ gas. A large sputtered ion yield, suggesting a surface Coulomb explosion, has been observed from insulators bombarded with very highly charged EBIT ions.
\end{abstract}

PACS number: $34.80 . \mathrm{Kw}$ 


\section{Introduction}

The electron beam ion trap (EBIT) was developed at the Lawrence Livermore National Laboratory (LLNL) ten years ago for $\mathrm{x}$-ray measurements of highly charged ions [1]. Since then EBIT research has expanded into several new, exciting, and unanticipated directions. At LLNL much of the new research has been enabled by the development of new facilities. These include the conversion of the original EBIT into the higher energy Super EBIT [2], the construction of a second EBIT with extracted ion capability [3], the development of a cryogenic Penning trap (dubbed RETRAP) for retrapping highly charge EBIT ions [4], and the build up of a facility for the study of ion-surface collisions [5]. Many different kinds of measurements can be carried out with these facilities, and it is now possible to produce and study any charge state of any element, including bare $\mathrm{U}^{2+}$ ions [6].

The configuration of the trap used in both the EBIT and Super EBIT is shown in Fig. 1. Ions are trapped within the electron beam by the space charge potential of the beam itself, which amounts to several volts from the center to the edge of the beam. In the axial direction, the ions are confined by voltages applied to the top and bottom electrodes, typically 10 to 100 volts. An electron beam current of up to $200 \mathrm{~mA}$ is squeezed to a density of $\sim 5000 \mathrm{~A} / \mathrm{cm}^{2}$ by a 3-T magnetic field. The trap operates at a temperature of $4 \mathrm{~K}$, which reduces the density of background gas to an extremely low level. Ions are initially injected into the trap in low charge states from a vacuum spark source [7]; occasionally, neutral gas injection is used. The ions are stripped to very high charge states by beam electrons. Injection of a light gas, usually neon as shown in Fig. 1, 
is used in an evaporative ion-ion cooling process to compensate electron beam heating of the highly charged ions [8]. More information about EBIT operation can be found elsewhere [9].

This report summarizes recent results from four different EBIT research programs. Taken together, they are representative of current EBIT research at LLNL. Two of the experiments involve ( $\mathrm{x}$-ray or visible) spectroscopy of radiation from ions trapped in the LLNL Super EBIT. The other two experiments utilize highly charged ions extracted from the other EBIT at LLNL. In one experiment the ions are retrapped in a Penning trap, and in the other they are directed at a target surface.

\section{Ionization cross sections}

In a recent series of experiments, the Super EBIT at LLNL has been used to make the first direct measurements of the electron impact ionization cross sections for very highly charged ions. This was done with the geometry of Fig. 1 using $\mathrm{x}$-ray spectroscopy to measure the ionization balance of the trapped ions. K-shell ionization cross sections were obtained for a series of high-Z hydrogenlike ions $[6,10]$, and L-shell cross sections were obtained for uranium [11].

The equilibrium ionization balance of ions trapped in an EBIT is determined by the

relative rates of ionization and recombination. Thus, for hydrogenlike and bare ions the relationship between the cross sections and abundances at equilibrium is:

$$
\sigma_{H \rightarrow \text { bare }}^{\text {ion }}=\frac{N_{\text {bare }}}{N_{H}} \sigma_{\text {bare } \rightarrow H}^{R R}
$$


where $N_{b a r e}$ and $N_{H}$ are the abundances of the bare and hydrogenlike charge states. The cross sections for radiative recombination (the predominant recombination process) and ionization are given in obvious notation. The hydrogenlike ionization cross sections $\sigma_{\mathrm{H} \rightarrow \mathrm{bare}}^{\mathrm{ion}}$ can be obtained from Eq. (1) if the other quantities are known. The abundance ratios $\mathrm{N}_{\mathrm{barc}} / \mathrm{N}_{\mathrm{H}}$ were obtained from $\mathrm{x}$-ray spectra, and the radiative recombination cross sections were obtained from a relativistic atomic structure calculation [12].

Typical radiative recombination spectra for molybdenum and bismuth are shown in Fig. 2. They were accumulated for counting intervals of 14 and $35 \mathrm{sec}$, respectively, following a delay of several seconds after ion injection to allow the ionization balance to come to equilibrium. This cycle was repeated until sufficient statistics were obtained. Radiative recombination into the $\mathrm{K}$ shell of bare and hydrogenlike target ions produces a doublet of lines from which the abundance ratio of these ions was determined. The injection of neon cooling gas, required for evaporative cooling (see Fig. 1.), produces charge exchange recombination of the highly charged ions and modifies the ionization balance expected from Eq. (1). A correction for charge exchange was made by running at several different neon densities and extrapolating the observed ionization balance to zero neon pressure (at which point there would no longer be any highly charged ions in the beam).

The ionization balance technique was also used to obtain ionization cross sections for the $\mathrm{L}$ shell of uranium. In this case, the equilibrium abundances of $\mathrm{U}^{83+}$ (fluorinelike) through $\mathrm{U}^{89+}$ (lithiumlike) ions were obtained from L-shell radiative recombination spectra such as that shown in Fig. 3. Least squares fits were used to extract the intensities of the 
blended radiative recombination lines, whose energies are (fortunately) known to within a few $\mathrm{eV}$.

The effect of first order QED (i. e., the Moeller interaction as opposed to the static Coulomb interaction) in electron impact ionization has been explored in a recent relativistic distorted wave calculation [13]. This calculation is compared to the Super EBIT measurements for hydrogenlike molybdenum and bismuth in Fig. 4. The measured cross sections favor the theory with the Moeller interaction, particularly at higher electron energy where the different calculations diverge.

\section{Hyperfine splitting in ${ }^{165} \mathrm{Ho}^{66+}$}

The ground state of hydrogenlike ions with nonzero nuclear spin is split by the hyperfine interaction. The best known case is that of atomic hydrogen itself; where the splitting corresponds to the $21-\mathrm{cm}$ line observed in radio astronomy. For highly charged ions the size of the hyperfine splitting is proportional to $\mathrm{Z}^{3}$ and falls in the visible region of the spectrum for several isotopes, depending on the size of the nuclear magnetic moment. The first measurement of the hyperfine splitting for a high- $Z$ hydrogenlike ion was accomplished with laser fluorescence of ${ }^{209} \mathrm{Bi}^{82+}$ ions stored in a ring [14]. A substantial Doppler shift correction was required due to the relativistic velocity of the circulating ions. The only other high- $\mathrm{Z}$ measurement was done with ${ }^{165} \mathrm{Ho}^{66+}$ ions trapped in the Super EBIT at LLNL [15]. In this case the Doppler shift is zero.

A prism spectrograph fitted with a CCD detector was used to detect the $5726-\AA$ light from the hyperfine transition in hydrogenlike holmium as shown in Fig. 5. The 70$\mu \mathrm{m}$ diameter of the Super EBIT electron beam serves as the entrance slit for the 
spectrograph. The stigmatic focus of the spectrograph produces an image that is resolved along the direction of the electron beam, allowing a separation between light produced from neon atoms, which intersect the electron beam at only one location (see Fig. 1), and light produced from trapped ions. Known lines from neutral neon were used for a wavelength calibration of the spectrograph. Only about $6 \%$ of the trapped holmium ions are in the hydrogenlike charge state, and the upper hyperfine level is populated predominantly by ionization of heliumlike ions. The signal from the $F=4$ to $F=3$ hyperfine transition in ${ }^{165} \mathrm{Ho}^{66+}$ obtained from 36 hours of data collection is shown in Fig. 6. The other spectrum in Fig. 6 was obtained with the electron beam energy set below threshold for production of $\mathrm{Ho}^{66+}$, and therefore below threshold for production of the hyperfine line. Conditions were otherwise identical, confirming the identification of the observed line as a transition in $\mathrm{Ho}^{66+}$. To minimize background light, all sources of light other than the unavoidable radiation from the electron gun cathode were eliminated, and longer wavelength light was blocked with a filter. Nevertheless, background spectra without trapped holmium were subtracted from the data to obtain each of the spectra displayed in Fig. 6.

The hyperfine splitting in a.hydrogenlike ion is proportional to the nuclear magnetic moment and includes small corrections for relativity, the distribution of nuclear magnetization, and QED. All of these quantities can be investigated with hyperfine splitting measurements such as this one. The measured splitting of $5726.4 \pm 1.5 \AA$ for ${ }^{165} \mathrm{Ho}^{66+}$ differs from a recent theoretical value [16] by $89 \AA$, a difference much larger than the QED correction. This theoretical value was calculated using the tabulated value of the 
${ }^{165} \mathrm{Ho}^{66+}$ magnetic moment. The theoretical value moves much closer to the experimental result if it is recalculated using a recent independent measurement of the ${ }^{165} \mathrm{Ho}^{66+}$ magnetic moment, suggesting that the present measurement is providing information primarily about the nuclear magnetic moment [15].

\section{Retrapped ions and charge exchange}

Ion traps have been widely used for many types of measurements, including collision studies. In some cases, multiply charged ions have been trapped and studied (see Ref. [17] and references therein). At LLNL, a recently completed cryogenic Penning trap (RETRAP) is being used to trap the very highly charged ions produced in an EBIT. A diverse research program is planned for this facility, including cooling and "freezing" of ions into crystals analogous to the core of white dwarf stars, laser spectroscopy of hyperfine transitions with minimal Doppler broadening, precision mass measurements, and charge exchange measurements. A recent measurement of charge exchange between very highly charged ions and $\mathrm{H}_{2}$ gas is described here [18].

A schematic layout of the RETRAP is shown in Fig. 7. It consists of two endcap electrodes, two compensation electrodes, and a center ring electrode. Ions are extracted from the EBIT in a short burst, decelerated, and captured by pulsing down the voltage on the top endcap. The axial motion of the ions is harmonic with an oscillation frequency proportional to the square root of the endcap potential:

$$
\omega_{2}=\left[q \mathrm{U}_{0} \mathrm{C}_{2} /\left(\mathrm{m} \mathrm{d}^{2}\right)\right]^{1 / 2}
$$


where $\mathrm{U}_{0}$ is the endcap potential, $\mathrm{C}_{2}$ is a constant, $\mathrm{q}$ and $\mathrm{m}$ are the ion charge and mass, and $d$ depends on the characteristic radial and axial dimensions of the trap through the relation $2 d^{2}=\left(z_{0}^{2}+r_{0}{ }^{2} / 2\right)$. The endcap potential is used to bring the axial oscillation frequency into resonance with a 1.2-MHz tuned circuit (see Fig. 7). This circuit has a Q of about 250 , and the initially hot ions cool to a temperature of a few $\mathrm{eV}$ with a characteristic time of roughly $0.8 \mathrm{sec}$. The signal from the tuned circuit, amplified with a low noise cryogenic preamplifier, can be used as an ion detector by ramping the endcap potential so that the different ion charge states pass through resonance.

The charge exchange cross section with $\mathrm{H}_{2}$ gas was measured with RETRAP for highly charged ions of xenon and thorium. Nondestructive ion detection using the ion's axial motion is sensitive enough to enable a single ion to be followed as it changes its charge. This is illustrated in Fig. 8, where a thorium ion, injected initially as $\mathrm{Th}^{79+}$ and observed at 10-sec intervals, changes its charge first to $78+$ and then to $77+$. Charge exchange rates were obtained from multiple observations of this type, and from runs in which many ions were trapped simultaneously so that the charge state distribution could be observed. The density of $\mathrm{H}_{2}$ molecules in the trap was calibrated from the observed charge exchange rate of $\mathrm{Ar}^{11+}$, for which the cross section has been previously measured by other methods [19]. Cross sections were measured for several charge states of both xenon and thorium. The results show that the charge exchange cross section increases linearly with charge to a value of approximately $2 \times 10^{-13} \mathrm{~cm}^{2}$ at $\mathrm{q}=79$ [18]. Double charge exchange (i.e., capture of both electrons from $\mathrm{H}_{2}$ ) was found to be about $25 \%$ of the total capture cross section. 


\section{Sputtered ions and Coulomb explosions}

Slow very highly charged ions are remarkable objects. They carry an enormous amount of potential energy that is of the same order or even larger than their kinetic energy. Such ions release their energy at the surface of the targets they hit, in marked contrast to fast ions, which penetrate the bulk material. Usually large numbers of electrons are removed from the target surface as a highly charged ion recombines. If the target is an insulator, or at least a material with low charge-carrier mobility, then a positively charged depletion region may form at the surface, as illustrated in Fig. 10. The characteristic time for recharging the depletion region, $\tau_{\sigma}$, is determined by the (nonlinear) conductivity of the material and may be of order $10^{-10} \mathrm{sec}$. The depleted region may gave rise to a so called Coulomb explosion, in which large numbers of atoms, ions, and clusters are ejected from the surface, and a shock wave propagates into the bulk material (see Fig. 10). The Coulomb explosion time is much shorter than the expected recharge time for the depletion region.

At LLNL the yield of ions sputtered from insulators was measured as a function of the charge of the incident ions [21]. Sputtered ions were identified and mass analyzed by their time of flight to a microchannel plate located near the target. For negative secondary ions, the large prompt burst of electrons emitted in ion-surface collisions provides a natural start signal for time-of-flight measurements. Total secondary ion yield measurements for $\mathrm{SiO}_{2}$ coated silicon targets are shown in Fig. 11. A dramatic threeorder-of-magnitude increase in the sputtered ion yield is indicative of the large surface energy release for highly charged incident ions. 
The spectrum of ions sputtered from a surface is another indication that something qualitatively different happens when highly charge ions hit a surface as compared to incident ions with low charge. Measurements at LLNL show a large yield of sputtered ion clusters of the form $\left(\mathrm{SiO}_{2}\right)_{\mathrm{m}}^{-}$for $\mathrm{Th}^{70+}$ ions incident on $\mathrm{SiO}_{2}$ surfaces at an energy of $9 \times \mathrm{q}$ $\mathrm{keV}$ [21]. These clusters are not observed at all for low charge incident ions; however clusters as large as $\mathrm{n}=10$ were observed for $\mathrm{Th}^{70+}$ incident ions. This threshold behavior of cluster emission supports a Coulomb explosion picture of sputtering for very highly charged ions.

\section{Acknowledgments}

I would like to thank my colleagues in the Livermore EBIT program whose work is presented here. This work was performed under the auspices of the U. S. Department of Energy by Lawrence Livermore National Laboratory under Contract No. W-7405Eng-48. 


\section{References}

[1] R. E. Marrs, M. A. Levine, D. A. Knapp, and J. R. Henderson, Phys. Rev. Lett. 60, $1715(1988)$.

[2] D. A. Knapp, R. E. Marrs, S. R. Elliott, E. W. Magee, and R. Zasadzinski, Nucl. Instrum. Methods. A $\underline{334}, 305$ (1993).

[3] D. Schneider et al., Phys. Rev. A 44, 3119 (1991).

[4] D. Schneider et al., Rev. Sci. Instr. 65, 3472 (1994).

[5] D. Schneider et al., Surface Sci. 294, 403 (1993).

[6] R. E. Marrs, S. R. Elliott, and D. A. Knapp, Phys. Rev. Lett. 72, 4082 (1994).

[7] I. G. Brown, J. E. Galvin, R. A. MacGill, and R. T. Wright, Appl. Phys. Lett., 49 , $1019(1986)$.

[8] M. A. Levine, R. E. Marrs, J. R. Henderson, D. A. Knapp, and M. B. Schneider, Physica Scripta T22, 157 (1988).

[9] R. E. Marrs, in Experimental Methods in the Physical Sciences Volume 29A, edited by F. B. Dunning and R. G. Hulet, (Academic Press, San Diego, 1995), p. 391.

[10] R. E. Marrs, S. R. Elliott, and J. H. Scofield, unpublished.

[11] Th. Stoehlker, S. R. Elliott, R. E. Marrs, and J. H. Scofield, unpublished.

[12] J. H. Scofield, Phys. Rev. A $\underline{40,} 3054$ (1989).

[13] D. L. Moores and K. J. Reed, Nucl. Instr. Meth. B 98, 122 (1995).

[14] I. Klaft et al., Phys. Rev. Lett. 73 , 2425 (1994). 
[15] J. R. Crespo, P. Beiersdorfer, D. W. Savin, and K. Widmann, Phys. Rev. Lett. 77 826 (1996).

[16] V. M. Shabaev, J. Phyș B 27, 5825 (1994).

[17] D. A. Church, Phys. Rep. 228, 253 (1993).

[18] B. R. Beck et al., Phys. Rev. Lett. 77, 1735 (1996); J. Steiger et al., Nucl. Instr. Meth. B 98,569 (1995).

[19] S. D. Kravis et al., Phys. Rev. A $\underline{52} 1206$ (1995).

[20] R. E. Olson and A. Salop, Phys. Rev. A 14, 579 (1976).

[21] M. A. Briere, T. Schenkel, A. E. Schach von Wittenau, and D. H. Schneider, unpublished; D. H. G. Schneider and M. A. Briere, Physica Scripta $\underline{53}, 228$ (1996). 


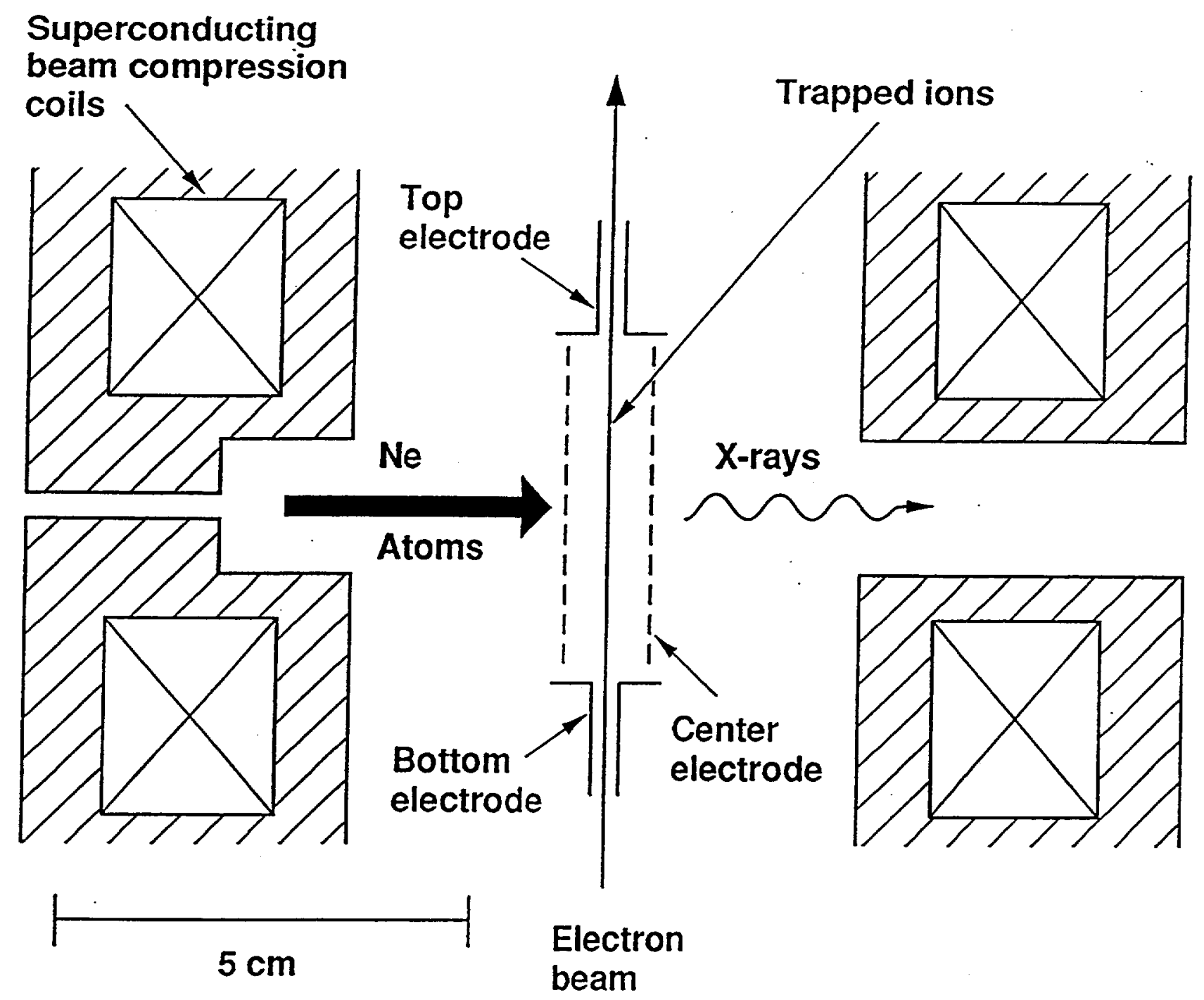

Fig. 1. Trap configuration for the EBIT and Super EBIT at LLNL. The cylindrical center electrode is slotted to enable measurements of $x$-ray and visible light emission. Neutral neon gas is injected for evaporative cooling. 

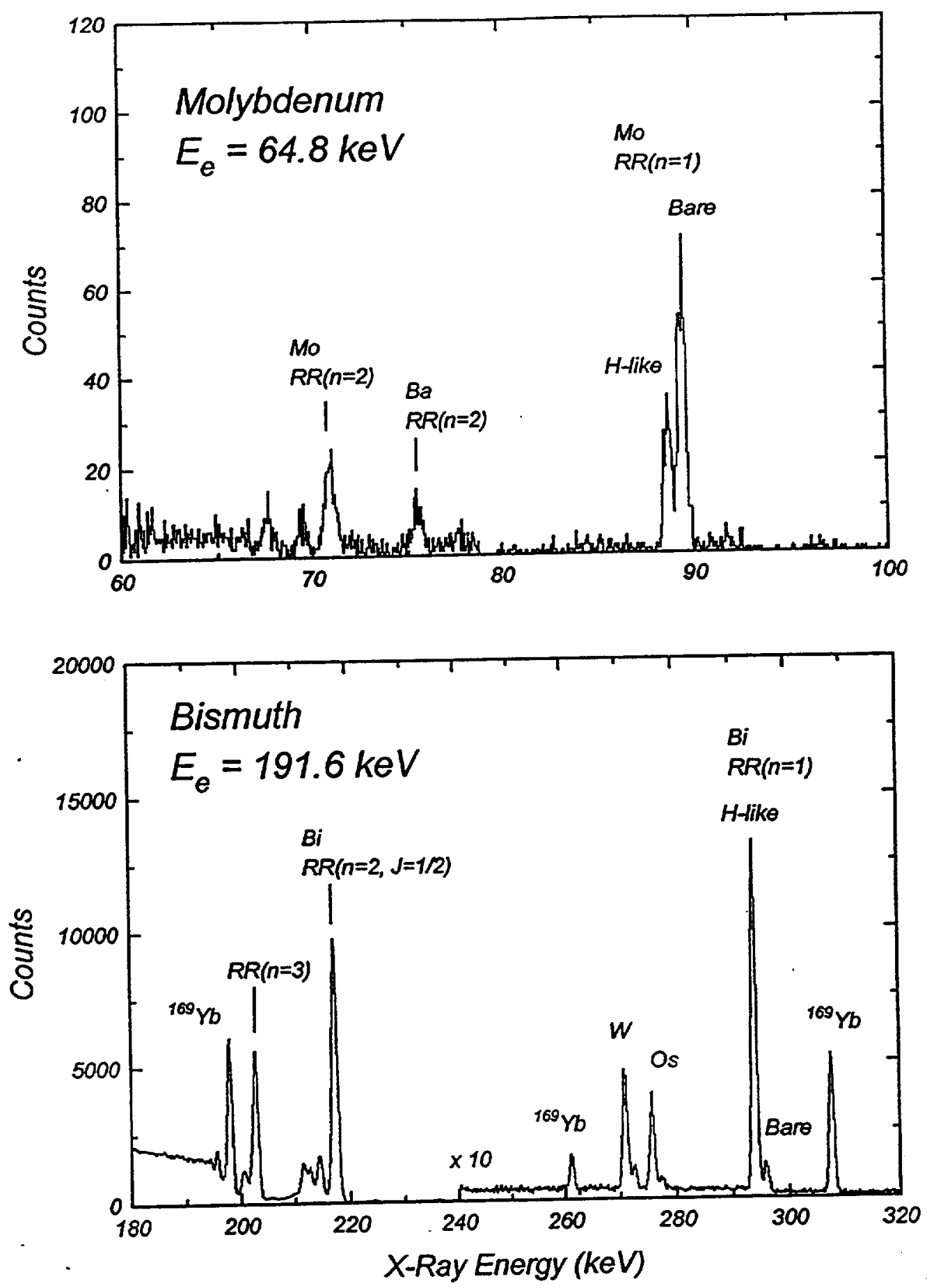

Fig. 2. Radiative recombination spectra obtained for molybdenum and bismuth. The $\mathrm{RR}(\mathrm{n}=1)$ doublet for H-like and bare target ions was used to obtain ionization cross sections for the hydrogenlike ions. In the bismuth spectrum the lines labeled ${ }^{169} \mathrm{Yb}$ are from a radioactive source used for energy calibration. Lines labeled $\mathrm{Ba}, \mathrm{W}$, and $\mathrm{Os}$ are from contaminant ions present in the trap. 


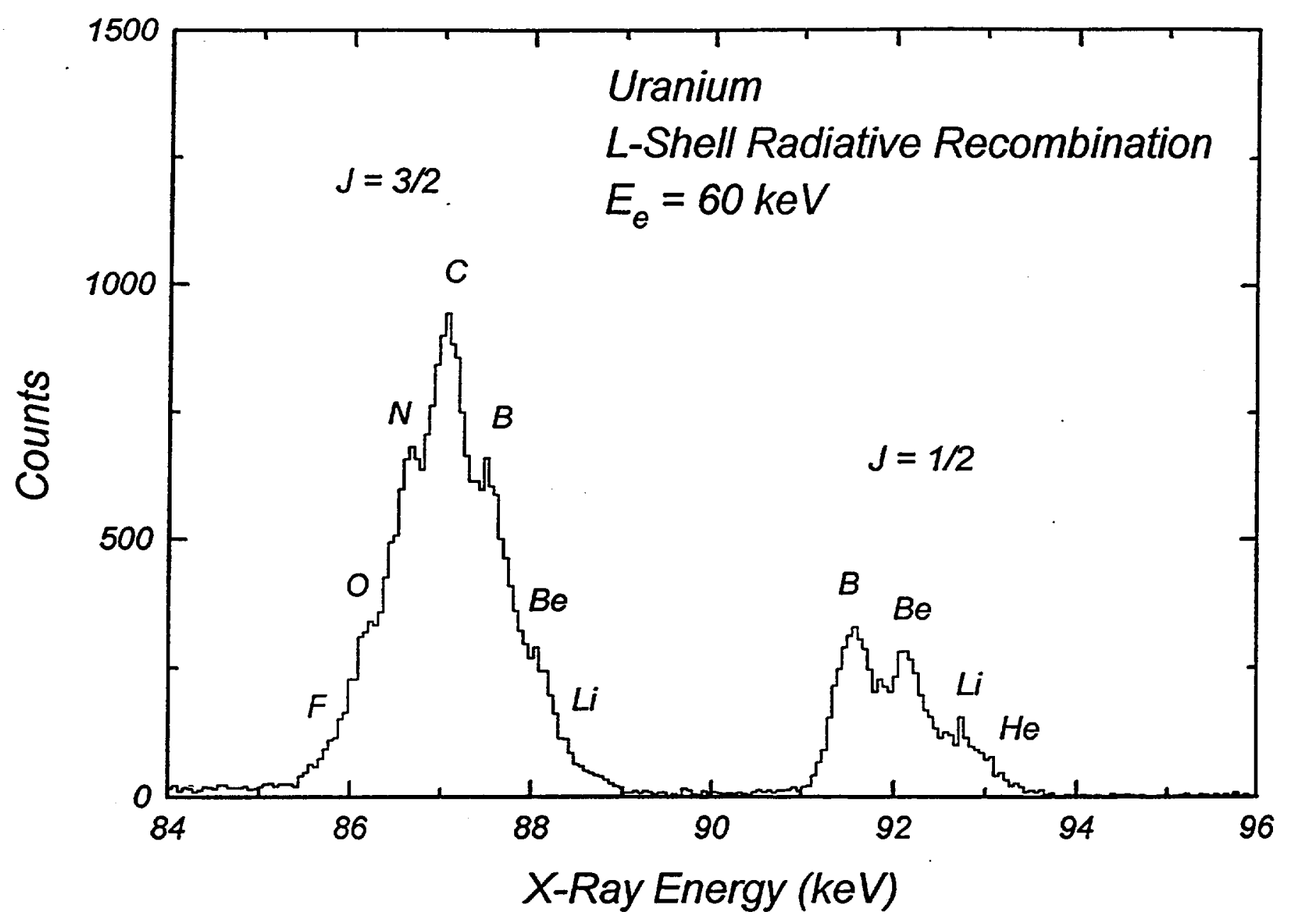

Fig. 3. L-shell radiative recombination spectrum for uranium ions at $60 \mathrm{keV}$. The approximate locations of the blended contributions of the different target-ion charge states are indicated by the chemical symbols. Some charge states contribute more than one line. 

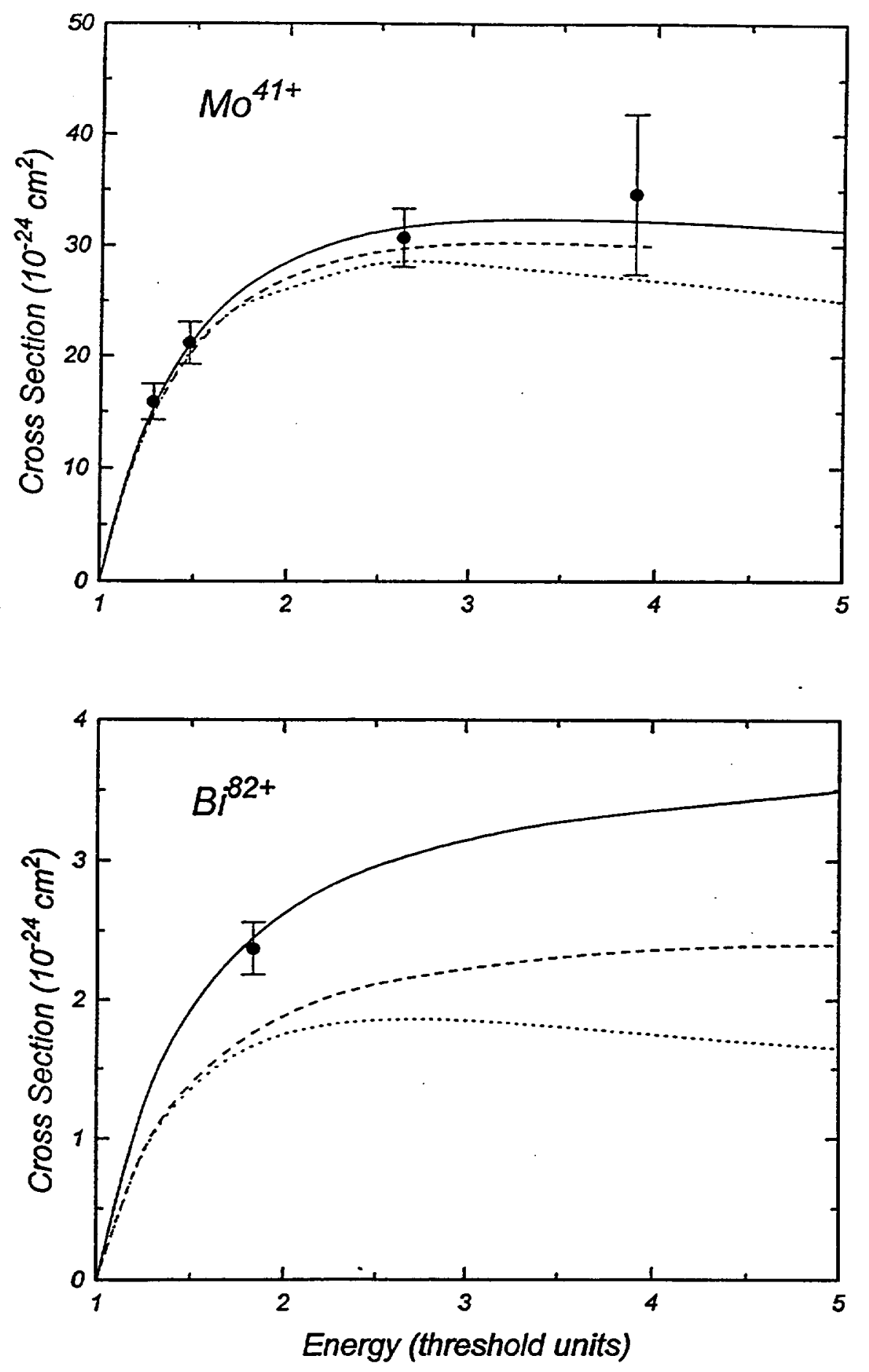

Fig. 4. The measured ionization cross sections for hydrogenlike molybdenum and bismuth compared to the relativistic distorted wave calculations of Ref. [13]. Solid curve: complete calculation including Moeller interaction. Dashed curve: relativistic calculation with Coulomb interaction only. Dotted curve: non-relativistic calculation. 


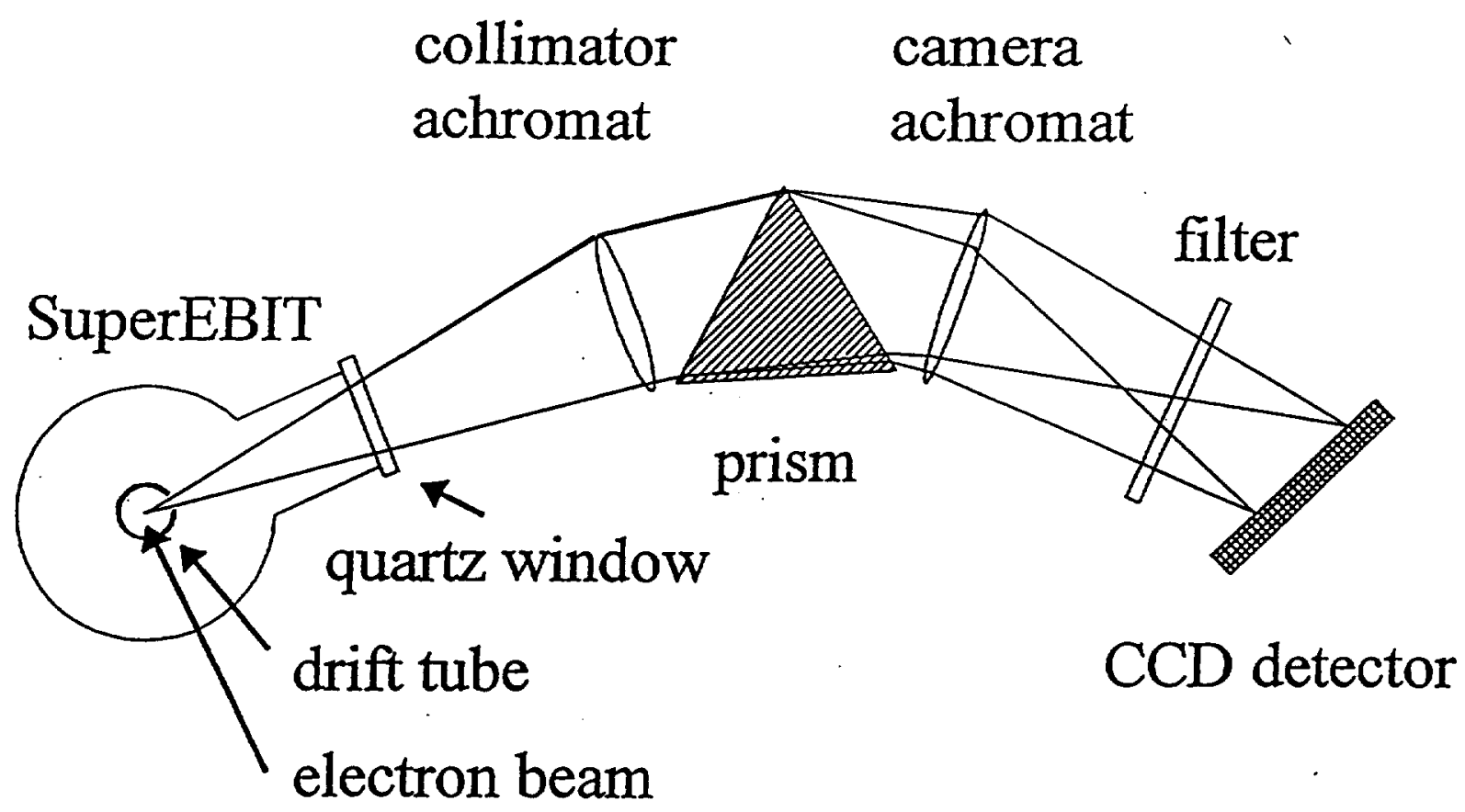

Fig. 5. Arrangement of the prism spectrograph used to observe the hyperfine transition in ${ }^{165} \mathrm{Ho}^{66+}$. 


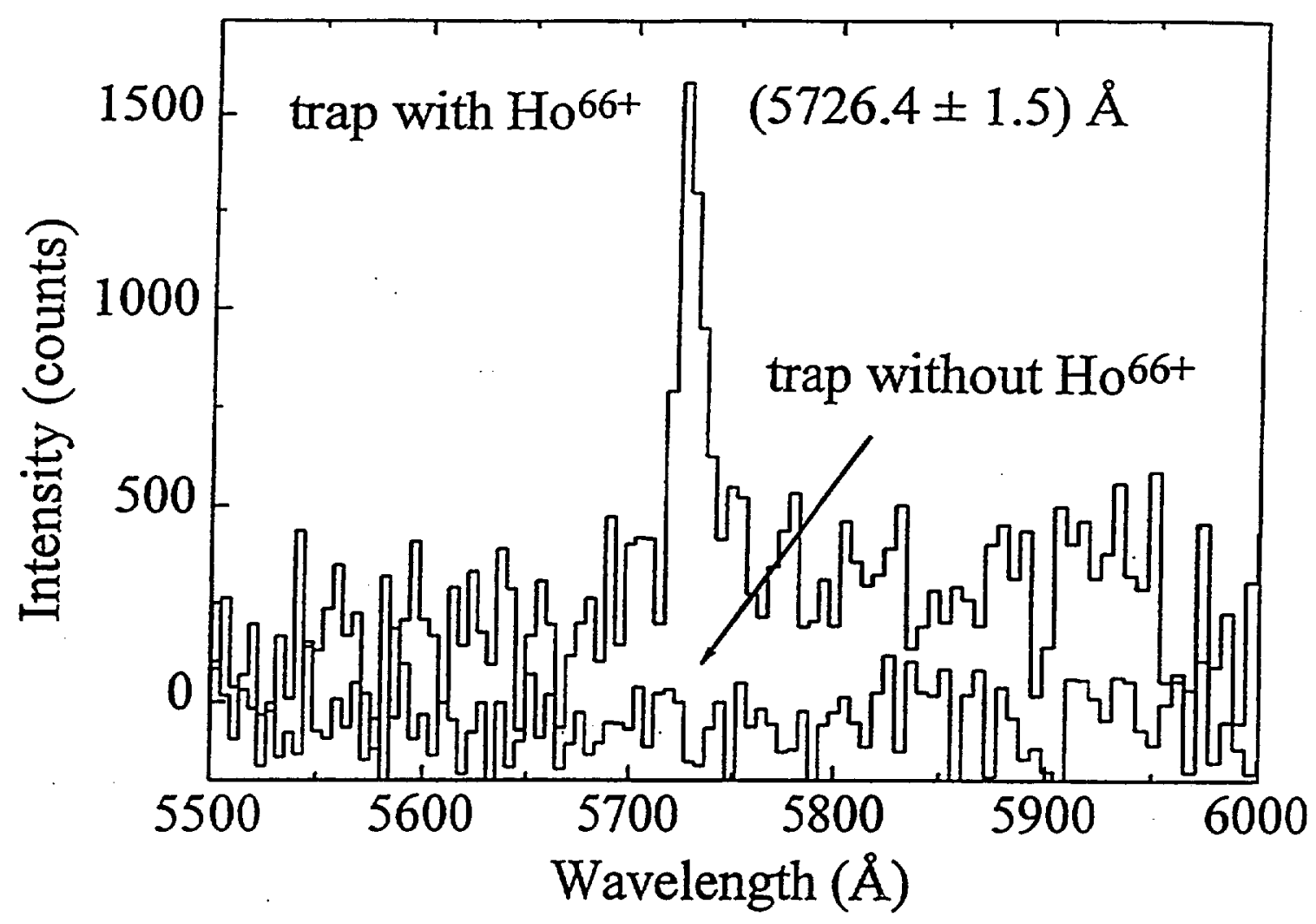

Fig. 6. The observed signal from the hyperfine transition in ${ }^{165} \mathrm{Ho}^{66+}$. The lower trace was obtained with the electron beam energy set below threshold for production of hydrogenlike holmium. 


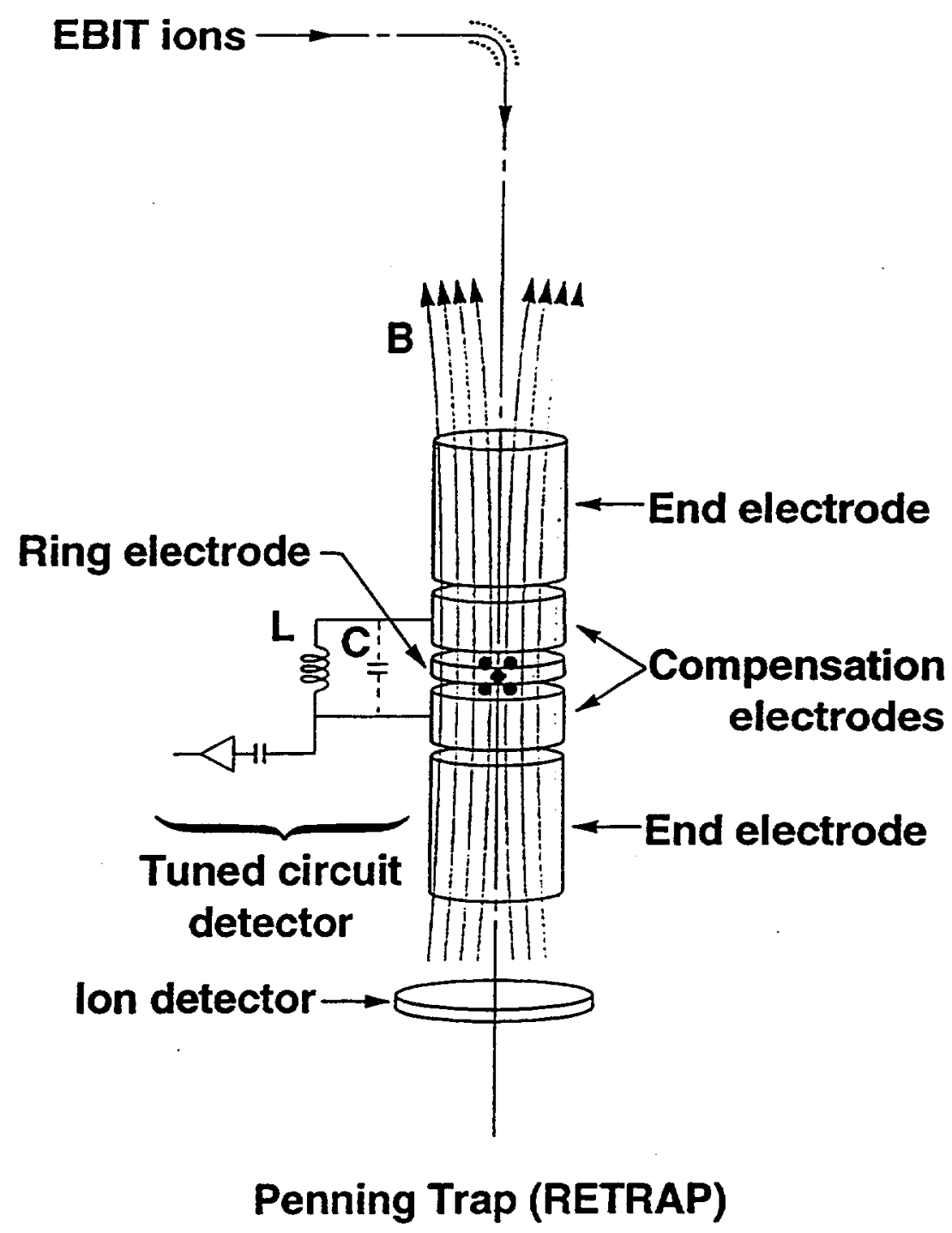

Fig. 7. Diagram of the Penning trap used for retrapping of highly charged EBIT ions. 


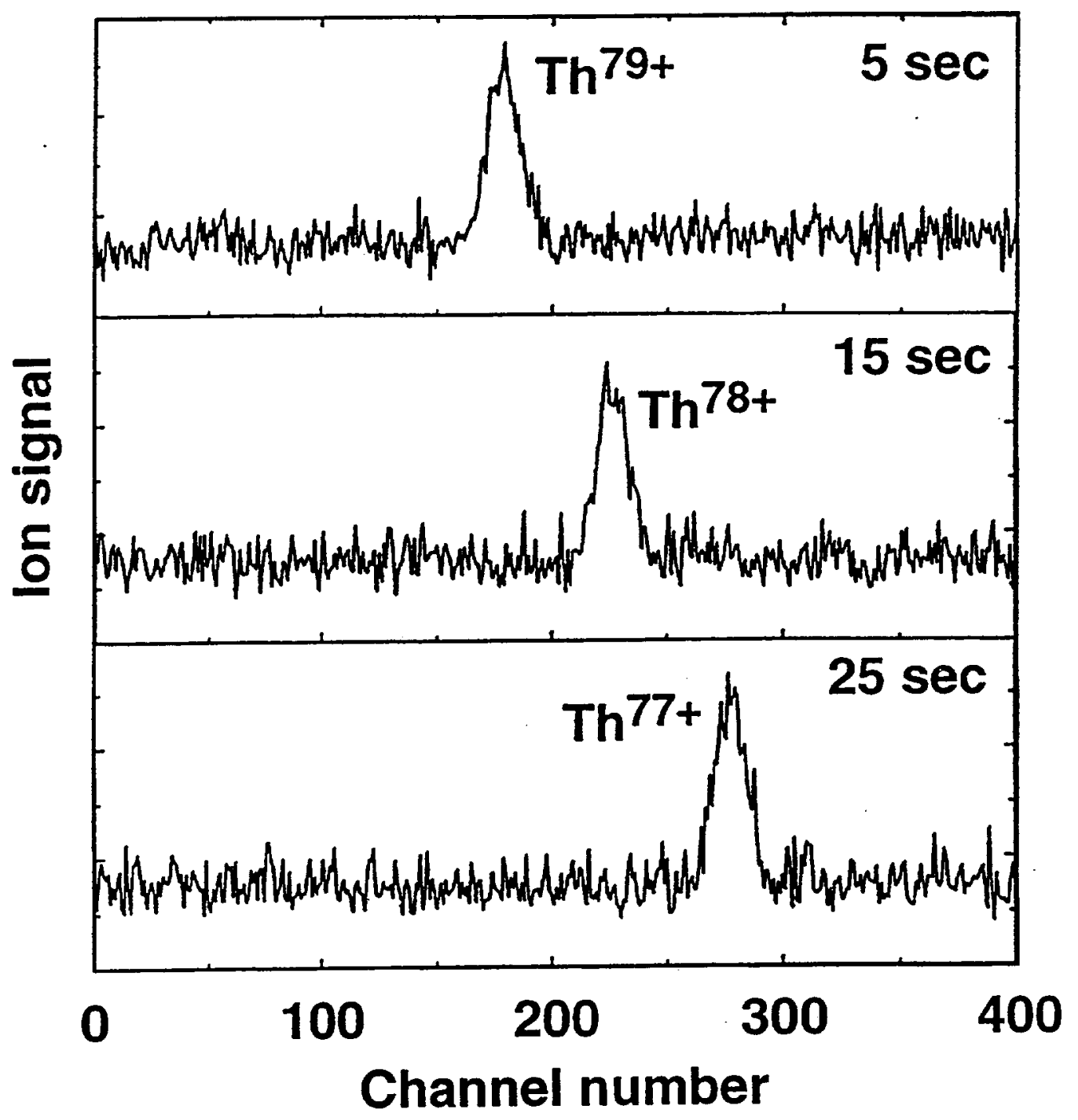

Fig. 8. Signal from the tuned circuit vs. endcap voltage (channel number) recorded at the indicated times. The peaks show sequential electron capture by a single thorium ion. 


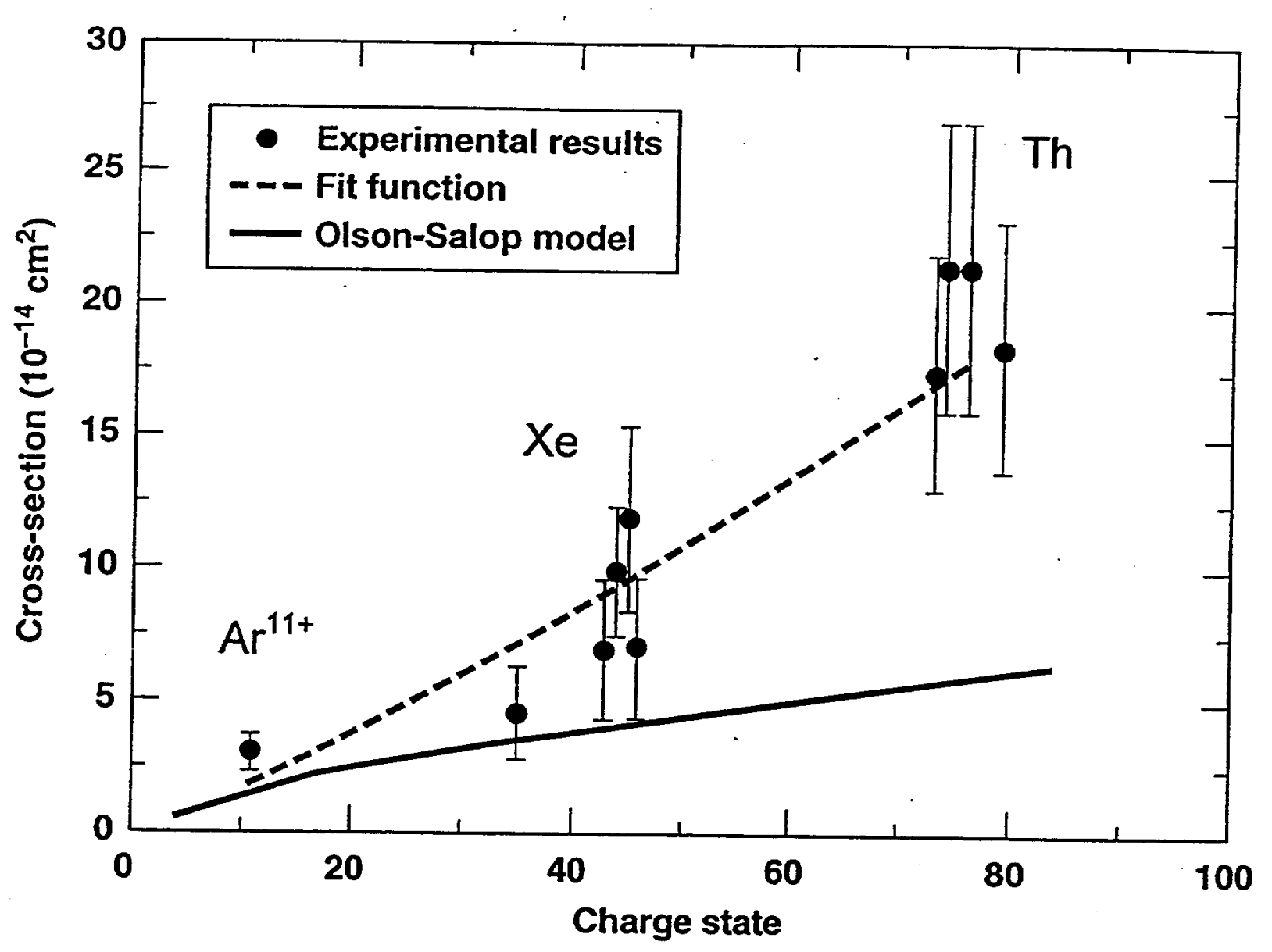

Fig. 9. Measured charge exchange cross sections for $\mathrm{H}_{2}$ gas. The $\mathrm{Ar}^{11+}$ point is from Ref. [19] and was used to calibrate the $\mathrm{H}_{2}$ density for the other points. The broken line is an empirical fit. The solid curve is an absorbing-sphere theoretical model [20]. 

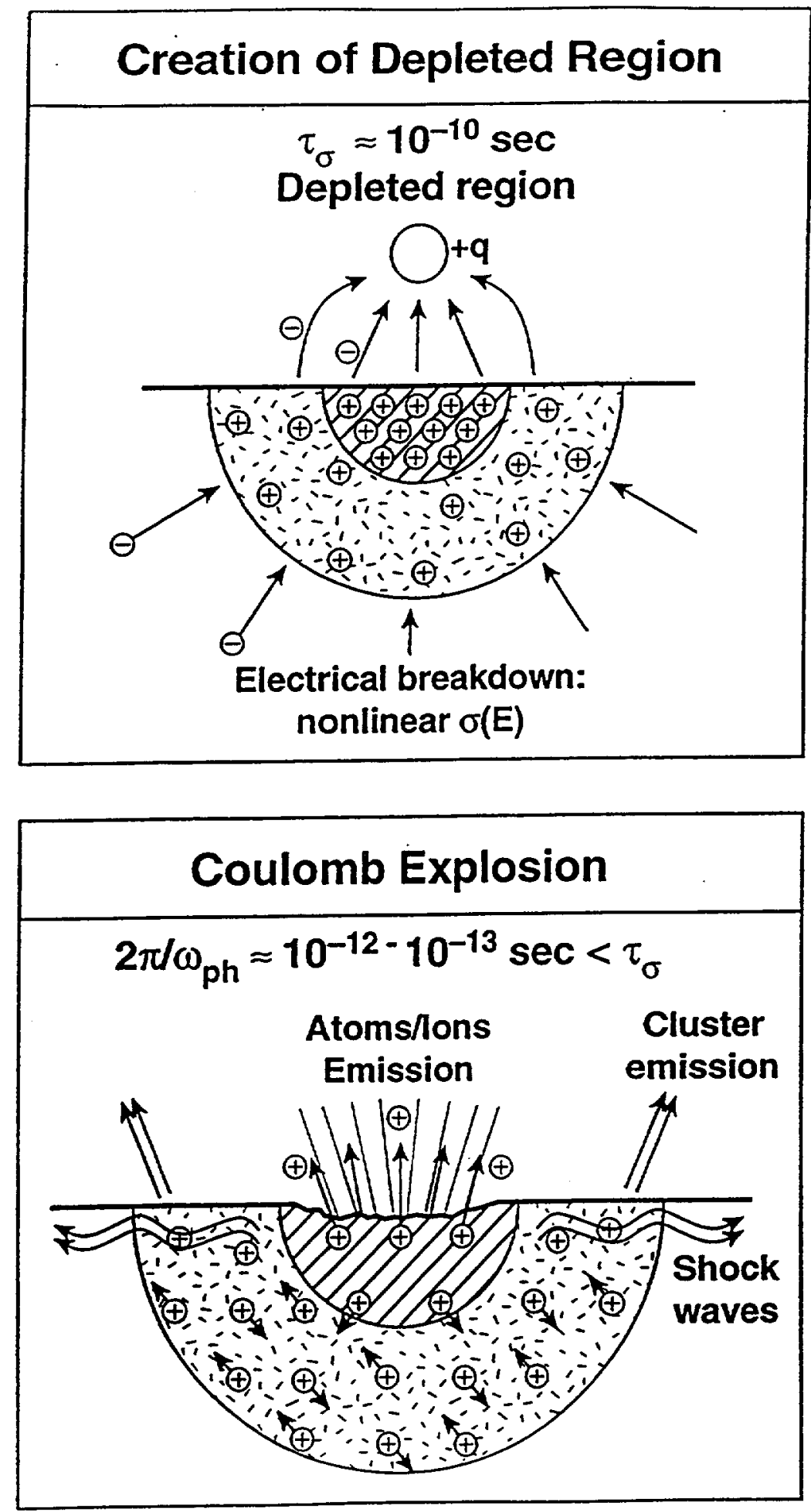

Fig. 10. Schematic of the Coulomb explosion process. Top: An incident ion removes electrons from the target faster than the characteristic time for replenishing them. Bottom: A Coulomb explosion occurs in the depleted region. 


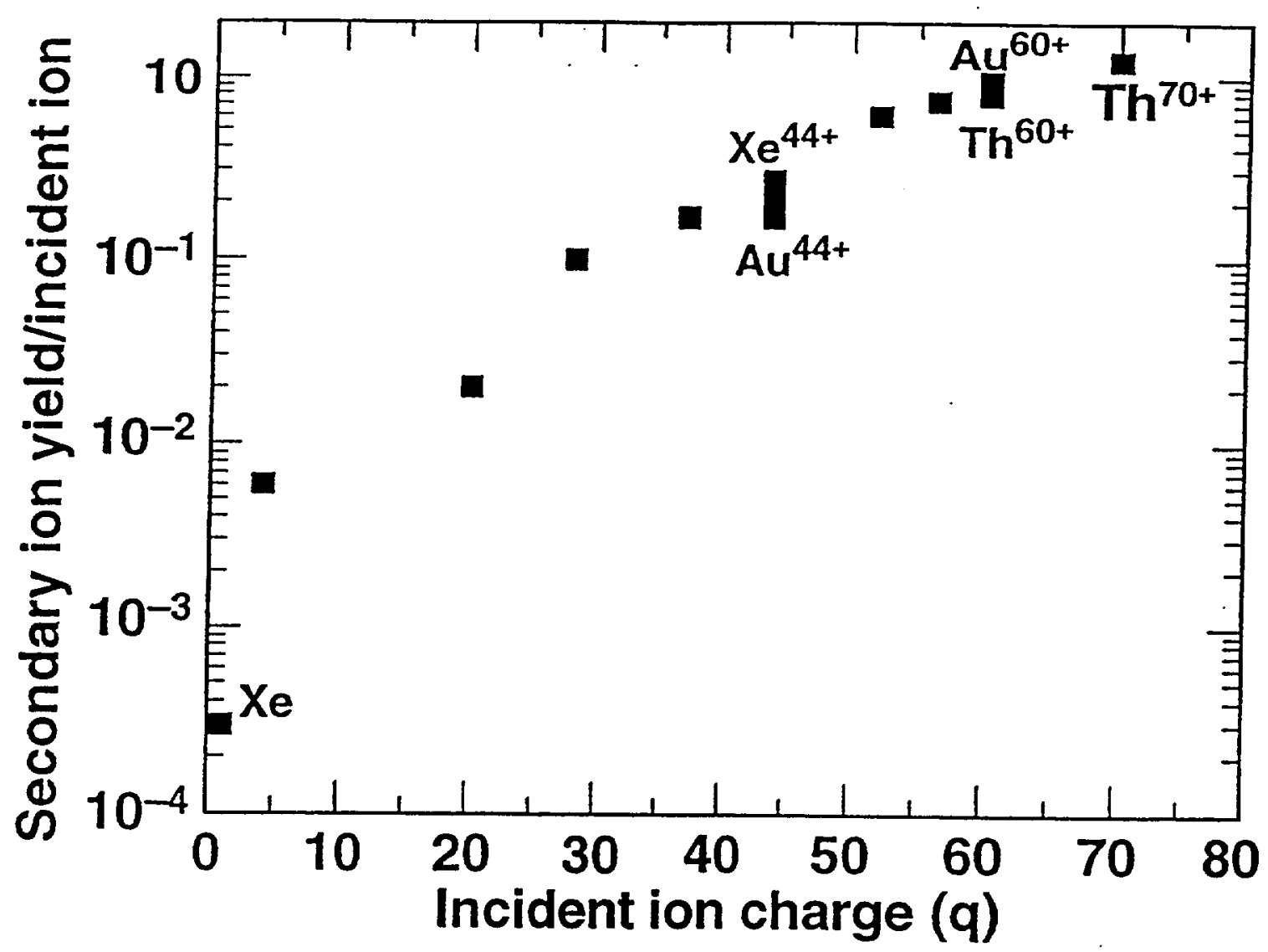

Fig. 11. Measured sputtered ion yield as a function of incident ion charge. 


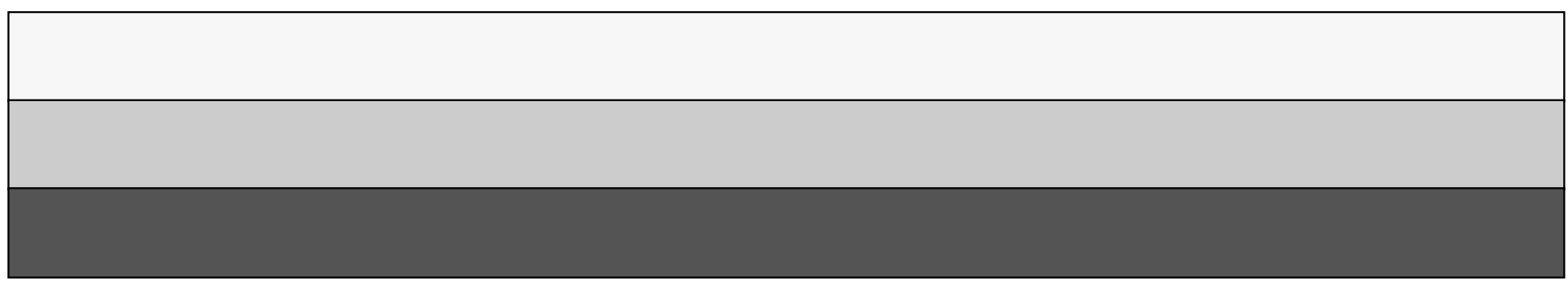

